\title{
Histone deacetylase inhibitor overrides the effect of soft hydrogel on the mechanoresponse of human mesenchymal stem cells
}

Rohit Joshi ${ }^{a}$, Pooja Dharmambal Murlidharan ${ }^{a}$, Pushpendra Yadava ${ }^{a}, V^{2}$ edanshi Dharnidharka ${ }^{a}$, and Abhijit Majumder ${ }^{\mathrm{a} *}$

${ }^{\mathrm{a}}$ Department of chemical engineering IIT, Bombay, 400076, India

*Corresponding author: abhijitm@iitb.ac.in

\begin{abstract}
:
Human Mesenchymal cells (hMSCs) are promising in regenerative medicine for their multi-lineage differentiation capability. It has been demonstrated that lineage specification is governed by both chemical and mechanical cues. Among all the different mechanical cues known to control hMSCs fate, substrate stiffness is the most well-studied. It has been shown that the naïve mesenchymal stem cells when cultured on soft gel, they commit towards adipogenic lineage while when cultured on stiff gel they become osteogenic. Soft substrates also cause less cell spreading, less traction, less focal adhesion assembly and stress fibre formation. Furthermore, chromatin condensation increases when cells are cultured on soft substrates. As the nucleus has been postulated to be mechanosensor and mechanotransducer, in this paper we asked the question how mechanosensing and mechanoresponse process will be influenced if we change the chromatin condensation by using an external chemical stimulus. To address this question, we treated hMSCs cultured on soft polyacrylamide (PA) gels with a histone deacetylase inhibitor (HDACi) called Valproic Acid (VA) which decondense the chromatin by hyperacetylation of histone proteins. We found that the treatment with VA overrides the effect of soft substrates on hMSCs morphology, cellular traction, nuclear localization of mechnosensory protein YAP, and differentiation. VA treated cells behaved as if they are on stiff substrates in all aspects tested here. Furthermore, we have shown that VA controls hMSCs differentiation via activation of ERK/MAPK pathway by increasing the p-ERK expression which inhibits adipogenic differentiation potential of mesenchymal stem cells. Collectively, these findings for the first time demonstrate that inhibiting histone acetylation can override the mechanoresponse of hMSCs. This work will help us to fundamentally understand the mechanosignalling process and to control the hMSCs differentiation in tissue engineering and regenerative medicine.
\end{abstract}

Key words: hMSCs, soft hydrogel, HDACi, p-ERK 


\section{Introduction:}

Substrate stiffness controls many different cellular behaviours such as cell morphology, maturation of focal adhesion, and formation of stress fibers $(1,2)$. Cells sense the mechanical properties of their microenvironment, by deforming the substrate via applying traction force. The information is then transduced to the nucleus via stress fibers and other mechano-transducing proteins (3-6). It has been observed, that on softer substrates the cells spread less, apply less traction, and the chromatin remains in a more condensed state. Recently, YAP (Yes-associated protein) and TAZ (transcriptional coactivator with PDZ-binding motif) the main transcriptional effector molecules of hippo signaling pathway have been identified as a key mechanosensor and mechanotransducers of mechanical cues $(7,8)$. Depending on substrate rigidity YAP/TAZ is known to shuttle between nucleus and cytoplasm. On soft substrates YAP translocates more into the cytoplasm (Inactivated) but on a rigid substrate, it localizes more in the nucleus (activated) and works as a transcriptional co-activator (8-10).

Not just the morphological changes, substrate rigidity also controls other critical cellular functions such as differentiation in the stem cells (11-13). It has been shown that human mesenchymal stem cells (hMSCs)favor adipogenic differentiation on soft substrate, while osteogenic differentiation is preferred on rigid substrates (14-16). Hence, understanding the mechano-signaling process is an active area of research in the field of regenerative medicines, stem cell biology, and tissue engineering.

Differentiation process is always linked with epigenetic modifications (17-19). During differentiation of hMSCs, genes responsible for self-renewal are turned off and genes related to lineage specifications are activated. Such controls are carried by various epigenetic changes including histone modifications. Acetylation of histones is one such important modification that makes the genes available for transcription by opening or decondensing the chromatin (20-22). Histone acetylation is controlled by two sets of modifiers, HATs (histone acetyltransferases) and HDACs (histone deacetylases). While HATs decondense the chromatin and make the genes transcriptionally more accessible by acetylating the histone proteins, HDACs work in the opposite manner by removing the acetyl groups from the histones and thus causing the chromatin to condense and making the genes inaccessible for transcriptional activities (23). In this context, it has been shown that a class of compounds known as histone deacetylase inhibitors ( $\mathrm{HDACi}$ ) which hyperacetylate histone proteins, promote osteogenic differentiation of hMSCs (24-28). However, there are a few contradictory reports as well showing a HDACi sodium butyrate stimulating adipogeneic gene expression and adipocyte differentiation (29, 30). A well-established HDACi called valproic acid (VA) has been shown to promote neuronal and hepatic differentiation of hMSCs (31-33). It also increases the anti-tumor effect of hMSCs in the gene therapy of glioma. Hence, it is important to understand the role of epigenetic modification such as 
histone acetylation/deacetylation in a specific context, mechanosensing being one. It is known that substrate rigidity controls hMSC differentiation and change chromatin packing (34). However, it is not known if chromatin reorganization via histone acetylation/deacetylation can overwrite the signals coming from substrates.

To fill this gap, we modified chromatin compaction of hMSCs with valproic acid $(\mathrm{VA})(35,36)$ when the cells were cultured on soft $(E=3 \mathrm{KPa}$ ) polyacrylamide (PAA) gels. It is known that soft substrate causes chromatin condensation and inhibits osteogenic differentiation (34). On the addition of VA, chromatin condensation decreases, as found by other researchers as well as by us (37). In this work, we have shown that on soft substrates when the cells were treated with VA, their chromatin decondensed. Concurrently, VA also modified many known effects of soft substrates on cellular morphology and functions such as cell spreading, cellular traction, expression of matured focal adhesion and stress fibres, nuclear localization of YAP, ERK phosphorylation, and differentiation. As soft substrates are known to promote adipogenic and supress osteogenic differentiation (38), in the presence of VA, this fate was reversed. Further, the level of p-ERK in the cell was found to increases upon addition of VA, which is known to promote osteogenesis (39). When phosphorylation of ERK was inhibited by using ERK pathway inhibitor PD 98059, the effect of VA was nullified. However, PD didn't have any effect on $\mathrm{CCP}$, in presence or absence of VA. Altogether, our work shows that acetylation status of chromatin overrides the effect of substrate stiffness via phosphorylation of ERK.

This work will be useful in understanding the mechanosignalling process fundamentally. It will also help us in controlling the cell fate by overriding the effect of microenvironment in tissue engineering and regenerative medicine. 


\section{Material and methods}

\section{Substrate preparation.}

Polyacrylamide gel having elastic modulus of $\sim 3 \mathrm{kPa}$ was prepared by crosslinking $40 \%$ poly-acrylamide and $2 \%$ bis-acrylamide solution. Protocol for substrate preparation and Elastic modulus value was adopted form previously reported work (40). Briefly, the gel solution for desired stiffness ( $3 \mathrm{kPa}$, Table S1) was mixed with ammonium per sulphate (1:100) and TEMED (1:1000) and a drop of 130 microliters was placed between two glass coverslips, one coated with 3-APTMS (Sigma) and other with hydrophobic coating. After polymerization, hydrophobic coverslip was removed. The gel was coated with type I collagen ( $25 \mu \mathrm{g} / \mathrm{ml}$ ) (Invitrogen; A1048301) using Sulfo-SANPAH based conjugation and kept at $4^{\circ} \mathrm{C}$ overnight (41).

\section{Cell culture}

Bone marrow-derived hMSCs were purchased from Lonza (Cat. No. \#PT-2501). hMSCs were cultured in Low glucose DMEM (Himedia; AL006) supplemented with 16\% FBS (Himedia ; RM9955), 1\% Antibacterial-Antimycotic (Himedia; A002) and 1\% Glutamax (Gibco; 35050) under humidified conditions at $37^{\circ} \mathrm{C}$ with $5 \% \mathrm{CO}_{2}$. The cells were trypsinized with TrypLE' (Gibco; 12604021) once they reached the confluence of $70 \%$. The cells were seeded on polyacrylamide gels with 2000 cells $/ \mathrm{cm}^{2}$ seeding density in $50 \mu$ of media and flooded after 45 mins.

\section{Treatment with HDACi}

To check the effect of HDACi on soft substrate $0.5 \mathrm{mM}$ of Valproic acid (PHR 1061) was added to the growth media at the time of flooding. For differentiation experiments, VA was added with differentiation media. For the experiments with Sodium Butyrate (SB, B5887), 0.5mM of SB was added in the similar manner.

\section{Differentiation assays}

hMSCs were seeded at 2000 cells $/ \mathrm{cm}^{2}$ in a 12 -well culture plate in growth medium for $24 \mathrm{~h}$ followed by differentiation media. Adipogenic (Invitrogen, A10410) and osteogenic (Invitrogen, A10069) differentiation kits were used. Cells were incubated for 9 days in adipo and 14 days for osteo induction media before quantitative assays. Differentiation media change was given after every third day. After the completion of differentiation duration, cells were fixed with 4\% PFA for $30 \mathrm{~min}$ at room temperature followed by staining with Oil Red O (Sigma 00625) for adipogenic differentiation and Alizarin Red (Sigma A5533) for osteogenic differentiation. After incubating with staining solution for 20 min samples are washed thrice with DPBS (adipo) or MilliQ (osteo). Images were captured for quantitative analysis using EVOS inverted microscope (Invitrogen) in bright-field colour channel. 


\section{Immunofluorescence staining}

Cells were fixed with 4\% paraformaldehyde (PFA) in PBS for $15 \mathrm{~min}$ at room temperature (RT) then washed with PBS thrice. Cells are then permeabilized with permeabilizing buffer (0.5\% Triton X-100Sigma Aldrich in CSB) for 10 min and blocked with BSA (4\% Bovine serum albumin in PBS) for 30 min to minimize nonspecific protein binding. Anti-YAP (1:500, rabbit, Abcam, Cat. No. 52771), anti-PPARY (1:500, rabbit, Abcam, Cat. No. 59256), anti-RUNX2 (1:500, rabbit, Abcam, Cat. No. 23981), anti-pERK (1:500, rabbit, Abcam, Cat. No. 65142), anti-OPN (1:500, rabbit, Abcam, Cat. No. 8448) primary antibodies in $4 \% \mathrm{BSA}$ were added to the samples and incubated overnight at $4^{\circ} \mathrm{C}$. Primary antibodies were removed and samples are rinsed with PBS two times for $10 \mathrm{~min}$. Samples were then incubated at room temperature with secondary antibodies (1:500, donkey anti-rabbit AlexaFlour 568 Cat. No. 75470, goat anti rabbit AlexaFlour 488 Cat. No. 411034) phalloidin (1:400, AlexaFlour 532, Cat. No. A22282 and AlexaFlour 488, Cat. No. A12379) with Hoechst 33342 (Cat. No. H3570) with dilution of 1:5000 in 4\% BSA for 2 hrs at room temperature. Afterwards secondary antibody solution was removed, and samples are rinsed two times with PBS. All immunostained samples were stored in PBS at $4^{\circ} \mathrm{C}$ until Imaging. All samples are imaged at $63 \mathrm{X}$ (oil) magnification using laser scanning Confocal Microscope (LSM, Carl Zeiss).

For vinculin staining hMSCs are fixed with ice cooled mixture of 1:1 (v/v) (4\% PFA: permeabilizing buffer (1\% Triton-X-100-Sigma Aldrich) for one minute on ice. Samples are rinsed with cytoskeleton stabilizing buffer (CSB) (60 mM PIPES, 27 mM HEPES, 10 mM EGTA, 4 mM magnesium sulphate, pH 7) and fixed again with 4\% PFA in ice for 5 min. After fixing, Samples are rinsed with CSB and blocked with $1.5 \%$ BSA supplemented with $0.5 \%$ Triton-X-100 for 30 min on ice. Anti-vinculin (1:500, rabbit Abcam Cat. No. ab129002, mouse monoclonal, Sigma) primary antibody in 4\% BSA were added to the samples and incubated overnight at $4^{\circ} \mathrm{C}$. Primary antibody was removed and samples are rinsed with PBS two times for $10 \mathrm{~min}$. Sample was then incubated at room temperature with secondary antibody (1:500, goat anti rabbit AlexaFlour 488 Cat. No. 411034), phalloidin (1:400, AlexaFlour 532, Cat. No. A22282) with Hoechst 33342 (Cat. No. H3570) with dilution of 1:5000 in 4\% BSA for 2 hrs at room temperature. All immunostained samples are imaged as previously mentioned.

\section{Traction force microscopy (TFM)}

Gels of $3 \mathrm{kPa}$ were made on $22 \times 22 \mathrm{~mm}^{2}$ coverslips, once gels were solidified, $25 \mu \mathrm{l}$ drop of $3 \mathrm{kPa}$ solution having $1 \mu \mathrm{m}$ fluorescent beads (Fluka with a final concentration of 1:50) was added on the hydrophobic plate and then the solidified gel was inverted into it and allowed to solidify. Gels are then treated with Sulfo-SANPHA and coated with collagen as mentioned above. Cells were seeded with the 
bioRxiv preprint doi: https://doi.org/10.1101/2022.01.04.474891; this version posted January $4,2022$. The copyright holder for this preprint

(which was not certified by peer review) is the author/funder, who has granted bioRxiv a license to display the preprint in perpetuity. It is made available under aCC-BY-ND 4.0 International license.

seeding density of 1000 cells/well. After $24 \mathrm{~h}$ of cell seeding, cells were lysed using $100 \mu$ l of $1 \%$ Triton$X$ in $2 \mathrm{ml}$ of complete media. Images of the stressed (before lysing) and unstressed (after lysing) gels were captured by the EVOS FL Auto cell imaging system (Invitrogen). An average of 10 cells was analysed per gel. The code from J. P. Butler was used to calculate the traction force.

\section{Statistical analysis}

Data are presented as means \pm standard deviation (otherwise mentioned) and were analyzed using Microsoft Excel software. Data was plotted using OriginLab software (IIT Bombay License). Statistically significant differences were claimed at: ${ }^{*}=p<0.05$. 


\section{Results}

\section{Valproic acid increases spreading of hMSCs cultured on soft substrates}

To explore the effect of chromatin modification on the mechanoresponse of hMSCs, we cultured the cells on soft polyacrylamide (PAA) gel ( $E=3 \mathrm{kPa}$; Table S1), with and without valproic acid (VA) $(0.5 \mathrm{mM})$ for the period of 24 hours. Valproic acid is a histone deacetylase inhibitor (HDACi) which inhibits histone deacetylase enzyme (HDAC) promoting hyperacetylation of chromatin resulting into chromatin decondensation. The concentration of VA was selected after confirming no considerable cell death at that concentration (data not shown).

Differential spreading of cells depending on substrate rigidity is one of the first observable mechanoresponse in hMSCs. They spread less on the substrates with low elastic moduli $(13,42)$ Hence, we checked the effect of VA on the spreading of hMSCs when cultured on the soft gels. We found that upon addition of VA, the cell spreading on $3 \mathrm{kPa}$ gels increased significantly ( $50 \%)$ compared to the control as shown in the fig 1 . We have also observed that in the presence of VA, cells show more protrusions (data not shown). To confirm that the effect is not VA specific, we used another histone deacetylase inhibitor sodium butyrate (SB) and found the similar outcome (fig. S1).
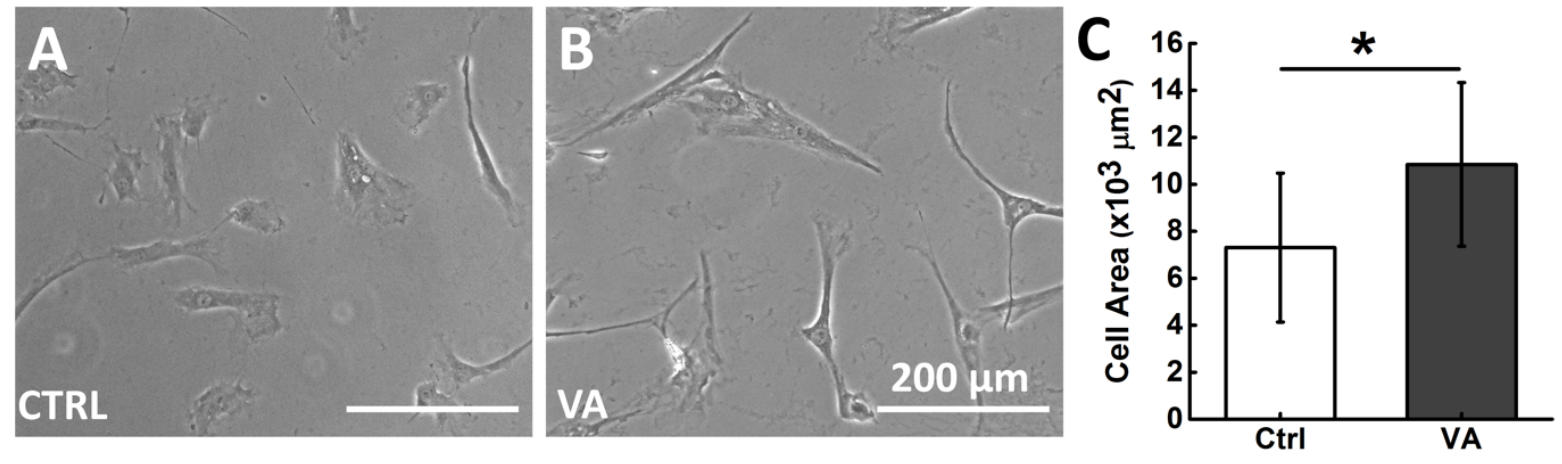

Fig 1. Effect of HDACi on projected cell area of hMSCs cultured on soft hydrogel: Representative phase contrast images of hMSCs cultured on $3 \mathrm{kPa}$ gel (A) without VA and (B) with VA and (C) related quantification. ${ }^{*} \mathrm{p}<0.05, \mathrm{~N}=3, \mathrm{n}=100$, scale bar: $400 \mu \mathrm{m}$.

\section{Valproic acid increases focal adhesion and actin stress fibre formation}

Cell spreading is known to be strongly associated with formation of matured focal adhesion and actin assembly (43). Hence, we examined the effect of VA on formation of focal adhesions (FAs) and actin stress fibre assembly by staining FA protein vinculin and F-actin respectively. Immunofluorescence images showed that cells treated with VA had significantly higher number of FAs (256 \pm 94$)$ as compared to control (94 \pm 40 ) (Fig $2 A, C, A$ \&C insets, \& E). The average area of FAs indicating 
maturation were also significantly higher in VA treated cells (Fig 2F). Similarly, we observed significantly higher amount and assembly of actin stress fibres when treated with VA (Fig 2B, D, G \& H), an atypical observation for cells cultured on soft substrates.

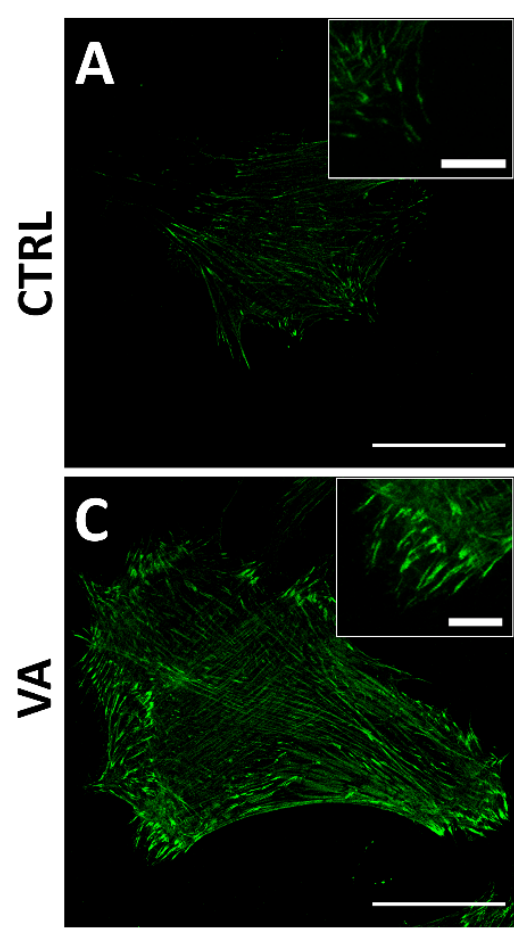

G
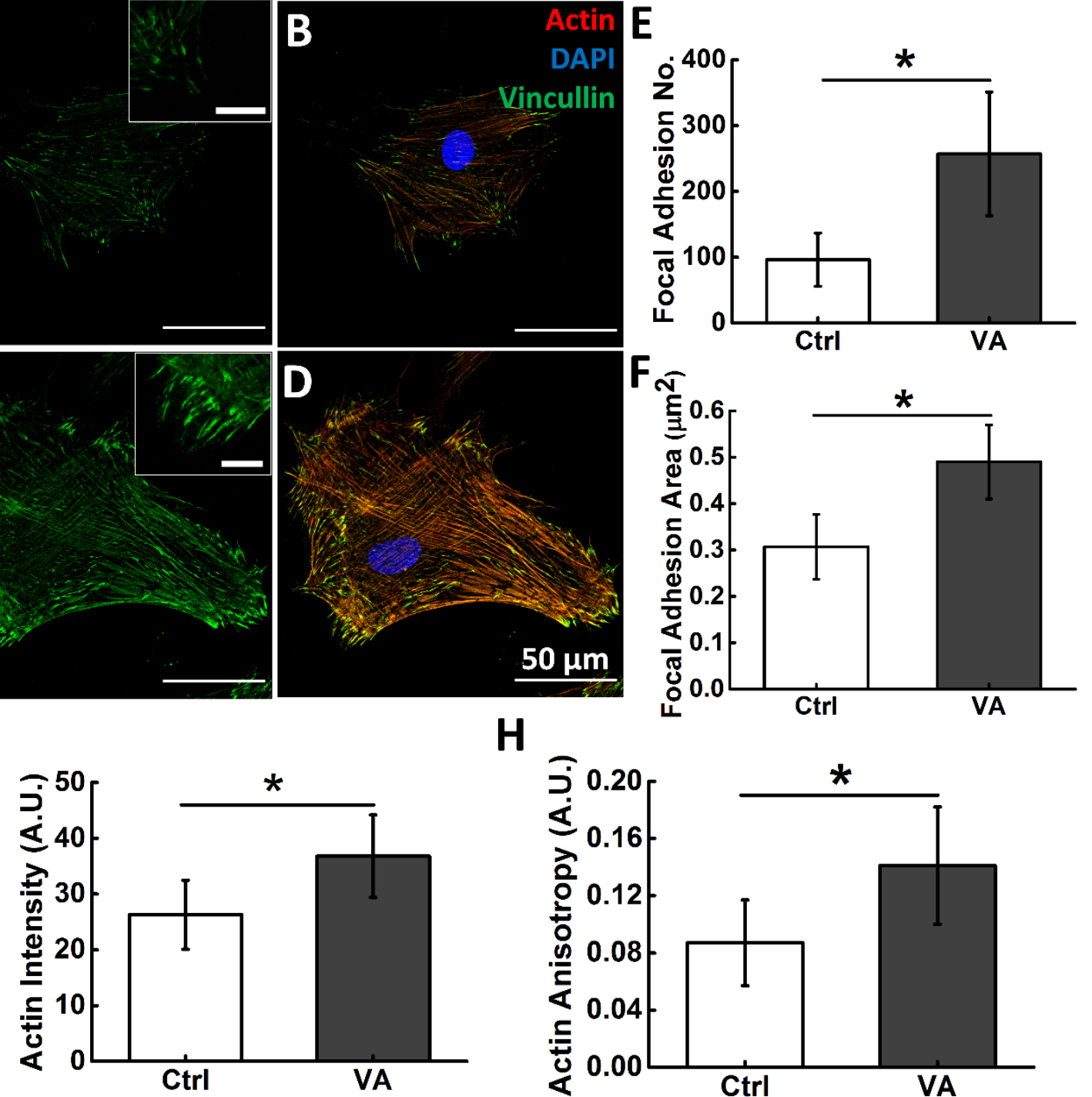

H

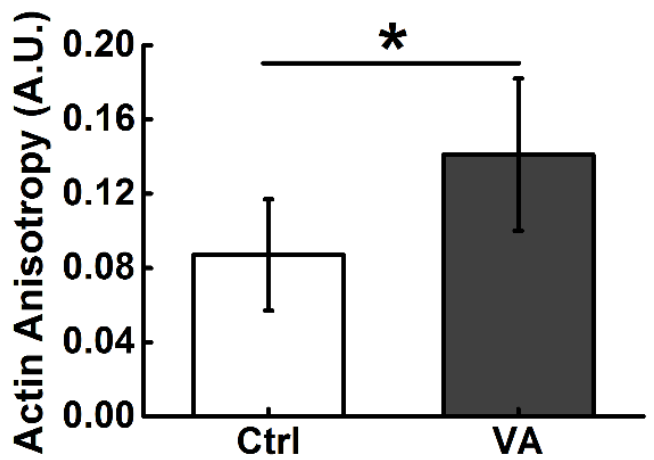

Fig 2. Effect of HDACi on focal adhesions of hMSCs cultured on soft hydrogel. Representative fluorescence images of vinculin (green) (A-D) and actin (red) (B and D) on $3 \mathrm{kPa}$ gel with (C and D) and without ( $A$ and B) Valproic acid. Graph E) shows change in focal adhesion no. with addition of VA with respect to control. and graph $\mathrm{F}$ ) shows change in focal adhesion area with addition of VA as compared to control. Graph ( $G$ \& H) shows change in actin intensity and anisotropy respectively with and without VA. ${ }^{*} p<0.05, N=2, n=16$. Scale Bar: $50 \mu m$. Inset Scale Bar: $5 \mu \mathrm{m}$ 


\section{HDACi increases cellular traction of hMSCs cultured on soft substrates}

From our earlier results, it was clear that HDACi treatment overrides the effect of soft gel $(3 \mathrm{kPa})$ on hMSC morphology, focal adhesion maturation and actin assembly. As all these cellular properties are strongly associated with cellular traction $(44,45)$, we investigated how HDACi and substrate rigidity together influence cellular contractility. We cultured hMSCs for $24 \mathrm{hrs}$ in PAA hydrogel ( $E=3 \mathrm{kPa})$ embedded with fluorescent beads (Fig. 3A). Subsequently, traction forces are calculated using Traction force microscopy (TFM). TFM revealed that VA significantly increases the traction force generated by hMSCs cultured on soft substrate (from $\sim 152 \mathrm{~Pa} \pm 32.05$ to $\sim 235 \mathrm{~Pa} \pm 39$ ) where they are known to be less contractile (44) as shown in the fig 3B-D. We also compared the interrelationship between cell spread area and traction. We found the cell spreading and traction holds a positive correlation as shown by others (46-48) for both with and without VA, albeit with different Pearson correlation coefficient ( 0.69 for control and 0.47 with VA), as shown in figure $3 \mathrm{E}$. We used another histone deacetylase inhibitor sodium butyrate (SB) and found the similar result (fig. S2). 


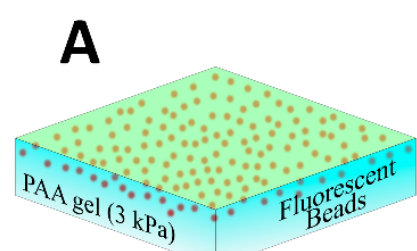

Relaxed gel

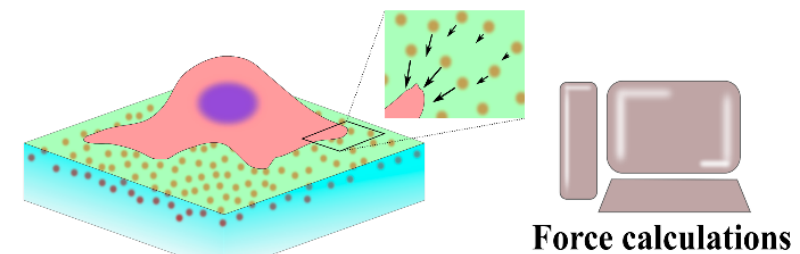

Stressed gel
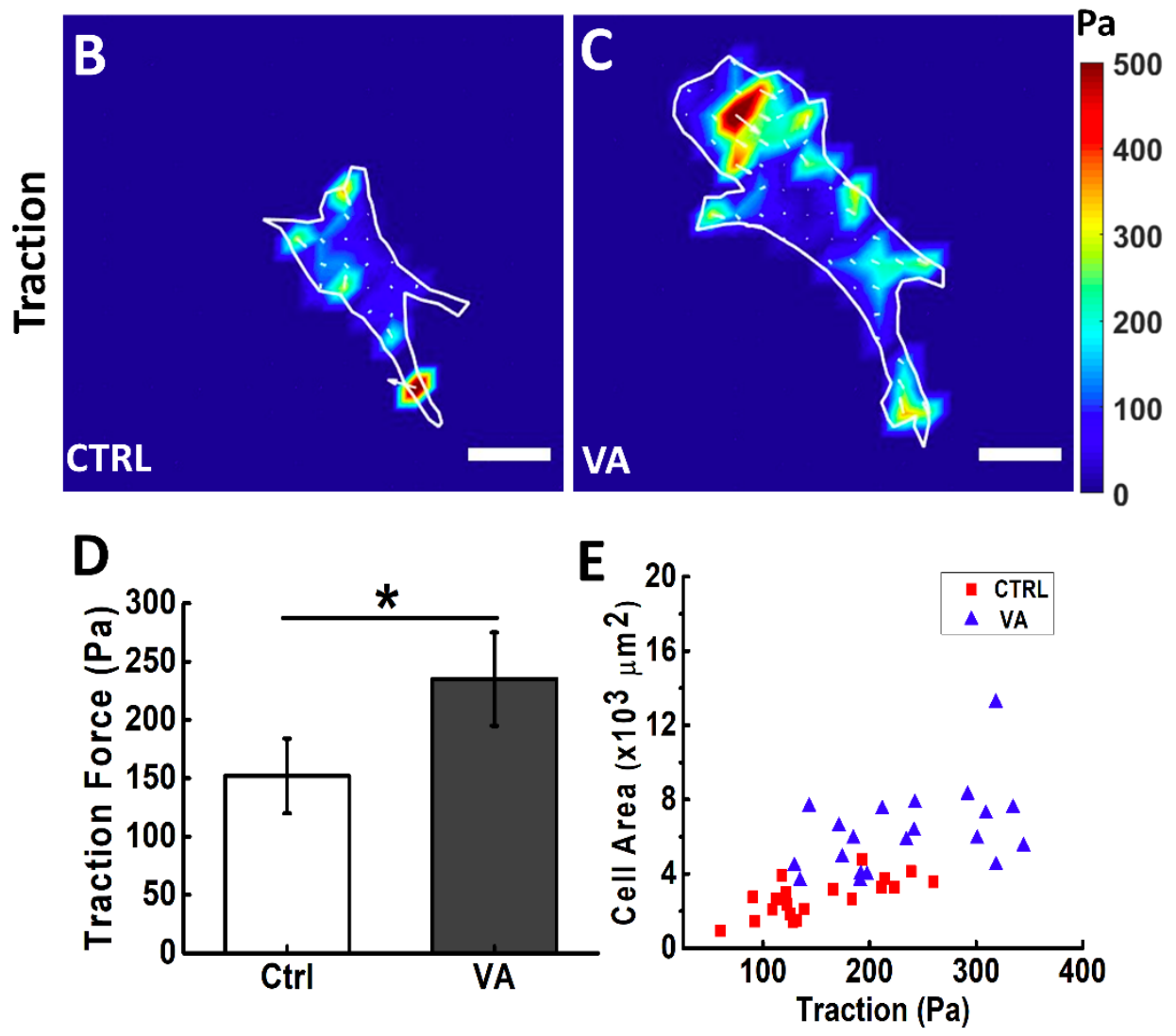

Fig 3. HDACi increase hMSCs traction on soft hydrogel. A) Schematic for PAA hydrogel-based TFM platform embedded with $1 \mu \mathrm{m}$ diameter fluorescent beads. Heat map of hMSCs traction force B) without VA, C) with VA. Colours correspond to magnitudes of forces as indicated in the colour bar. Graph D) shows change in traction force in addition of valproic acid with respect to control. E) shows positive correlation between cell spread area and cellular traction ${ }^{*} p<0.05, N=3, n>20$. scale bar : 20 $\mu \mathrm{m}$

\section{Effect of HDACi on YAP nuclear translocation of hMSCs cultured on soft substrates}

YAP (Yes-associated protein) is a cellular mechanosensor that translocates between nucleus and cytoplasm depending on substrate rigidity (8). This balance of nuclear to cytoplasmic ratio plays a crucial role in hMSCs fate determination. When hMSCs are cultured on a stiff substrate, YAP 
translocate more into the nucleus promoting osteogenesis while on the softer substrate, it localizes more in the cytoplasm promoting adipogenesis. As this is a key molecule in the mechanosensing process, it was imperative to check the effect of hDACi on substrate mediated YAP translocation. We found that when hMSCs were cultured on the soft gel $(3 \mathrm{kPa})$ in presence of valproic acid, nuclear localization of YAP increased by $\sim 1.5$ times as compared to control (Fig. 4 A-E). We also checked for nuclear to cytoplasmic ratio and found that in the presence of valproic acid nuclear to cytoplasmic ratio increased by 1.6 times as compared to control (Fig. 4F).

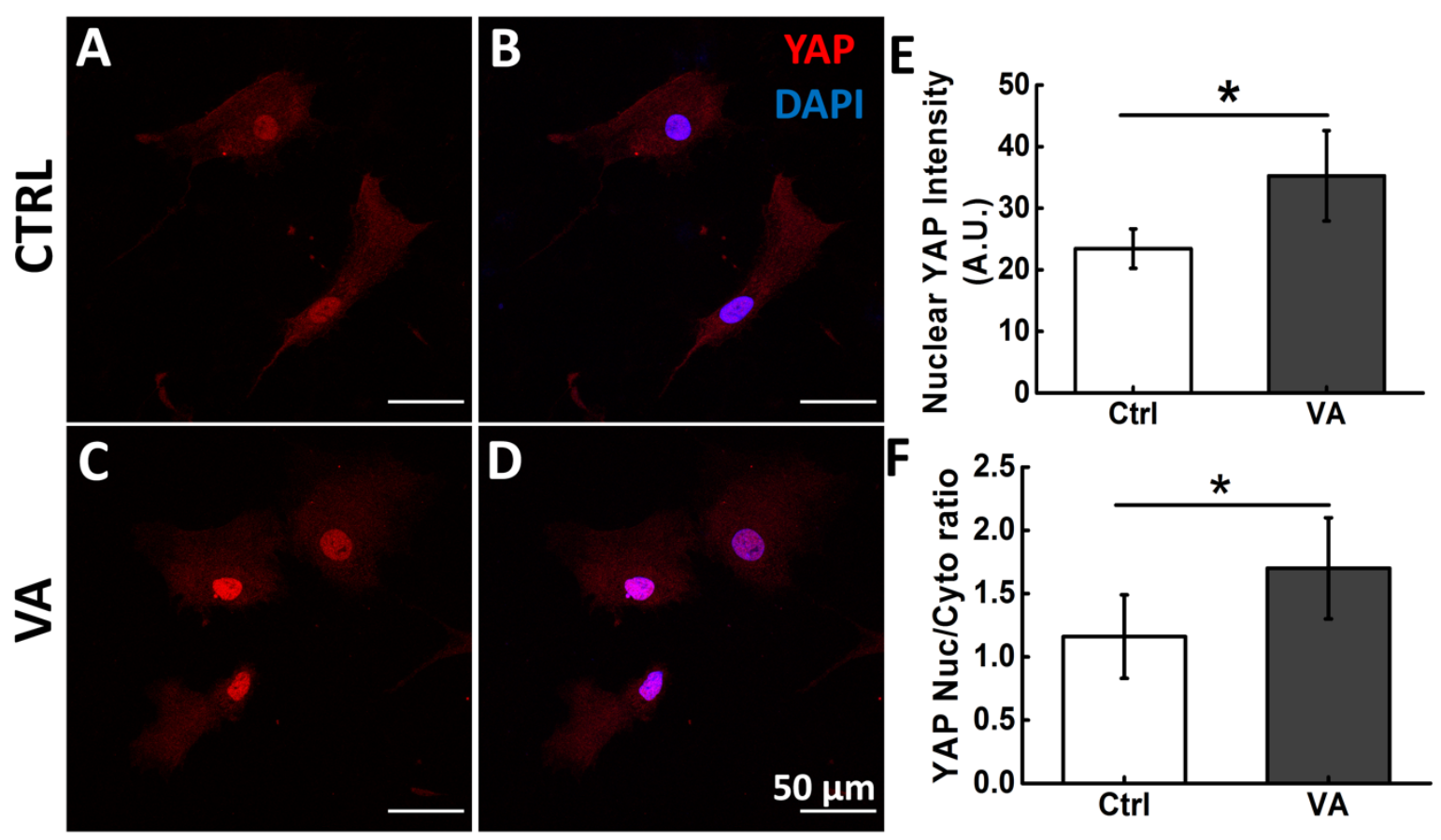

Fig 4. HDACi increases YAP nuclear translocation in hMSC cultured on soft hydrogel: Representative fluorescence images of YAP (red) and nucleus (blue) on 3kPa gel with (C \& D) and without (A \& B) Valproic acid. Graph E) shows change in Nuclear YAP intensity in addition to valproic acid with respect to control. (F) shows change in nuc/cyto ratio in addition to valproic acid with respect to control. ${ }^{*} \mathrm{p}<0.05, \mathrm{~N}=3, \mathrm{n}=30$, Scale Bar: $50 \mu \mathrm{m}$

Valproic acid suppresses adipogenic differentiation and promotes osteogenic differentiation of hMSCs on soft gel

One of the most important characteristics of hMSCs is their multilineage potential which makes them an attractive choice for tissue engineering. It has been widely reported that soft substrates promote adipogenic differentiation and suppress osteogenic differentiation of hMSCs (11-15). To explore the combinatorial effect of substrate stiffness and HDACi on adipogenic/osteogenic differentiation of 
hMSCs, we cultured them on collagen coated PAA gels of $3 \mathrm{kPa}$ stiffness with and without VA in presence of adipogenic/osteogenic differentiation media. We checked the formation of lipid oil droplets and expression of PPAR- $\gamma$ which are the markers for adipogenic differentiation. Similarly, to assess the osteogenic differentiation, we checked the expression level of RUNX2 and osteopontin which are the markers for osteogenic differentiation. We found that addition of VA reduces the formation of lipid oil droplets at 3kPa substrate stiffness by almost one third. (Fig. 5A-C). Similar results were observed from different HDACi i.e., sodium butyrate (Fig. S3). We also checked for PPAR- $\gamma$ which is a key transcriptional factor that regulates the expression of gene responsible for adipogenesis. We found that in the presence of VA PPAR- $\gamma$ expression decreases $\sim 28 \%$ compared to control. On the other hand, the expression of RUNX2 and osteopontin, two key transcriptional factors for osteogenesis, increases by $\sim 26 \%$ and $\sim 77 \%$ respectively in hMSCs cultured on soft gel in presence of VA as compared to control (Fig. 5G-L). These data together clearly shows that HDACi interferes with the mechanosignaling process in hMSCs and supersedes the effect of soft substrate on adipo/osteo differentiation. 

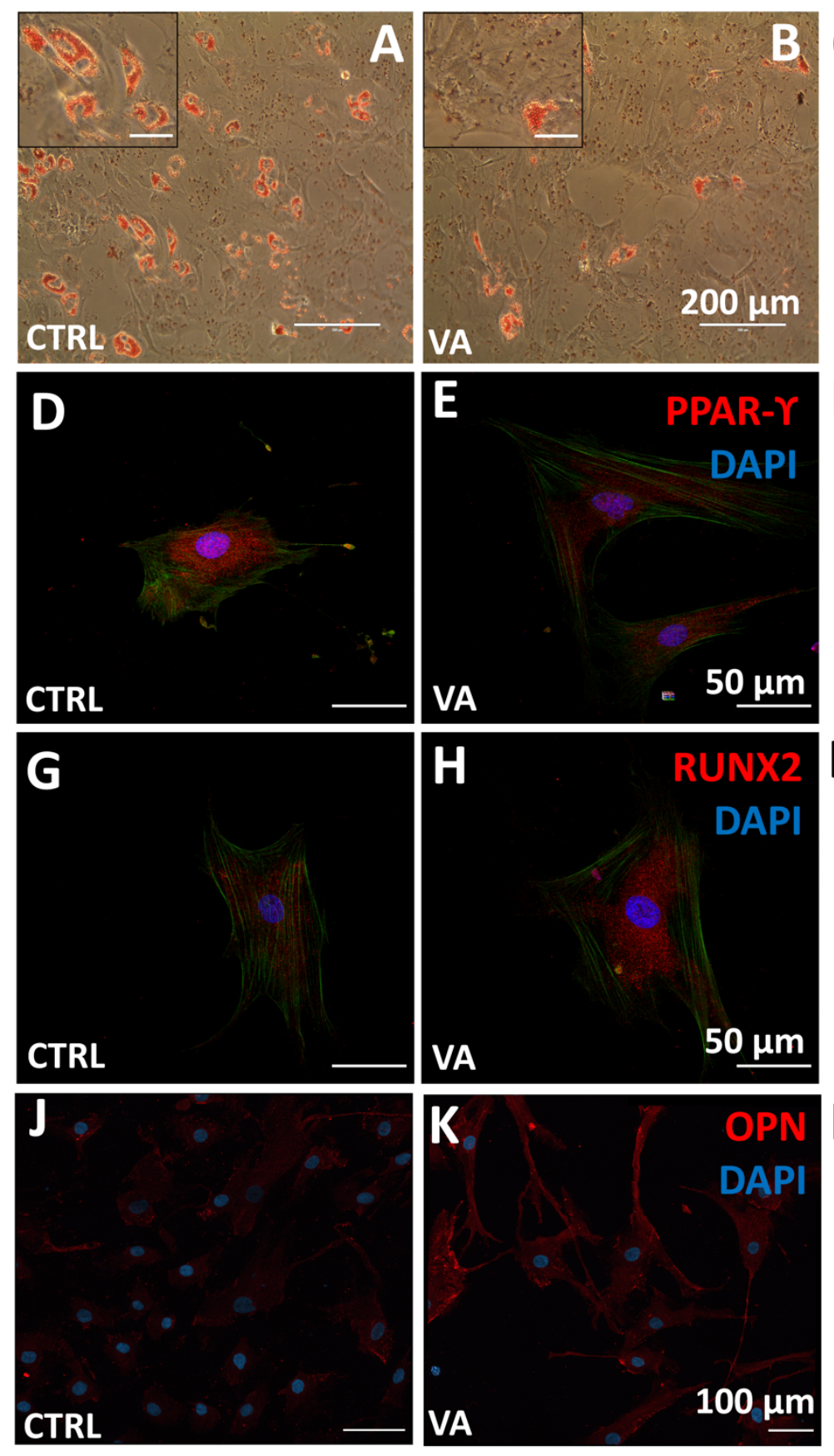
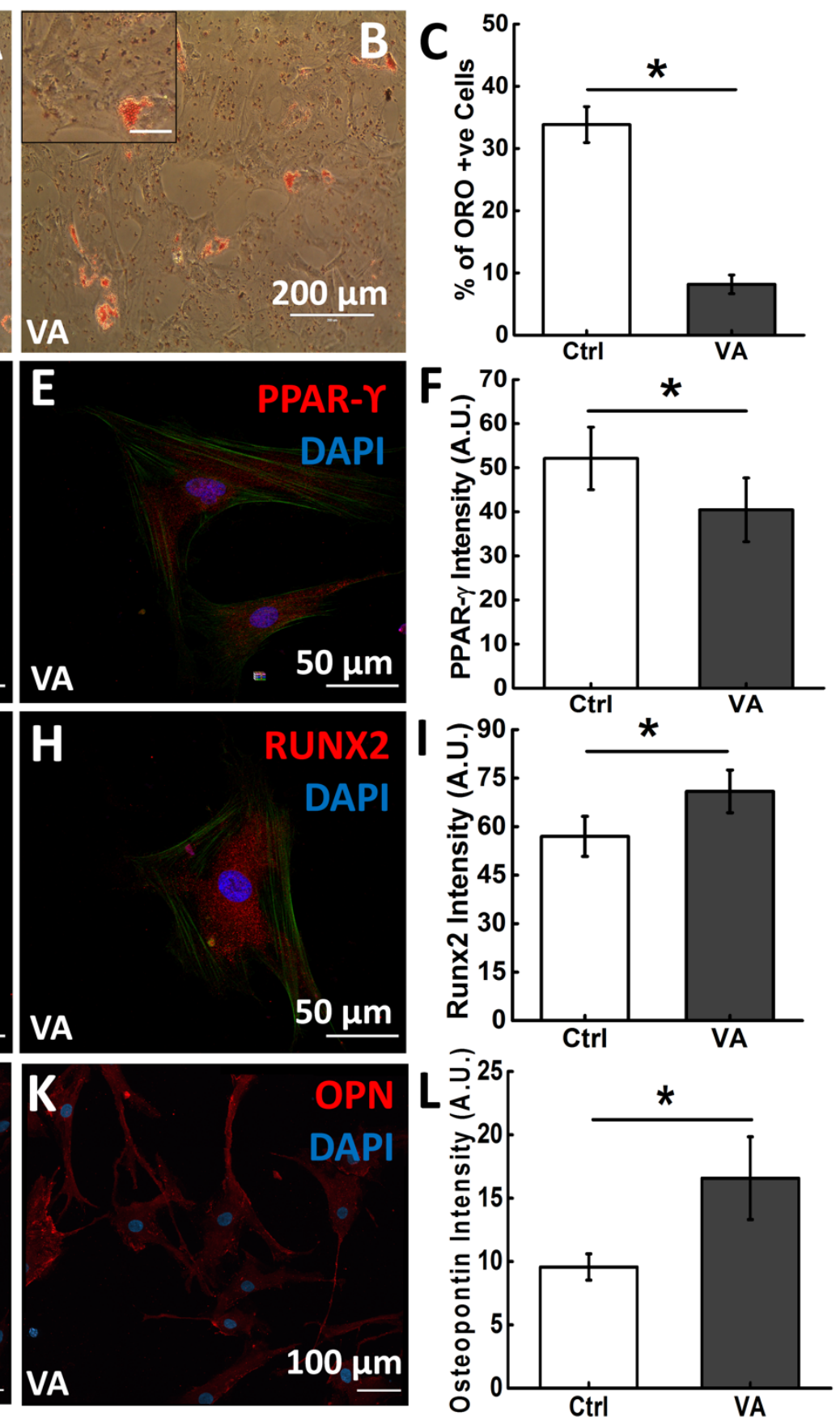

Fig. 5 HDACi inhibits adipogenesis of hMSCs cultured on soft hydrogels (3 kPA). Representative images of Oil red $O$ staining after 10 days culture in adipogenic media (A) without VA, and with VA (B). Graph (C) shows change in percentage of ORO positive cells with and without VA. Representative fluorescence images of PPAR-gamma (red) (D \& E) and Runx2 (red) (G \& H) on 3 kPa gel with and without VA. Graph F) and I) shows change in total PPAR-gamma \& RUNX2 intensity respectively with and without VA. Representative fluorescence images of osteopontin (OPN) with J) and without K) valproic acid. Graph L) shows change in osteopontin intensity in addition of VA with respect to control. ${ }^{*} p<0.05 \mathrm{~N}=3 \mathrm{n}>25$. 


\section{Effect of HDACi on p-ERK expression on soft substrate}

Our results suggest that in the presence of VA, adipogenic differentiation is inhibited even on compliant substrate. Earlier studies have shown that ERK/MAPK signalling plays a key role in regulation of mesenchymal stem cell differentiation (49). It is now known that stiff substrate promotes osteogenesis by activating ERK signalling pathway while soft substrate downregulates activated ERK Signalling pathway and promotes adipogenesis (50). Hence, we Investigated if VA overwrites the effect of substrate stiffness on osteo/adipo lineage specification via regulating ERK. We checked for the expression of p-ERK in the presence of VA by immunostaining and found it to be increased by 35 $\%$ as compared to the control (fig. 6A, B and E). To further investigate the essentiality of ERK activation

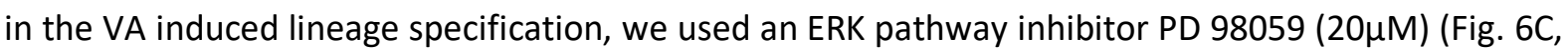
$D$, and E). Our results show that in the presence of PD 98059 there is an $\sim 25 \%$ decrease in the $p$-ERK expression. However, when VA and PD are used together p-ERK level is restored.

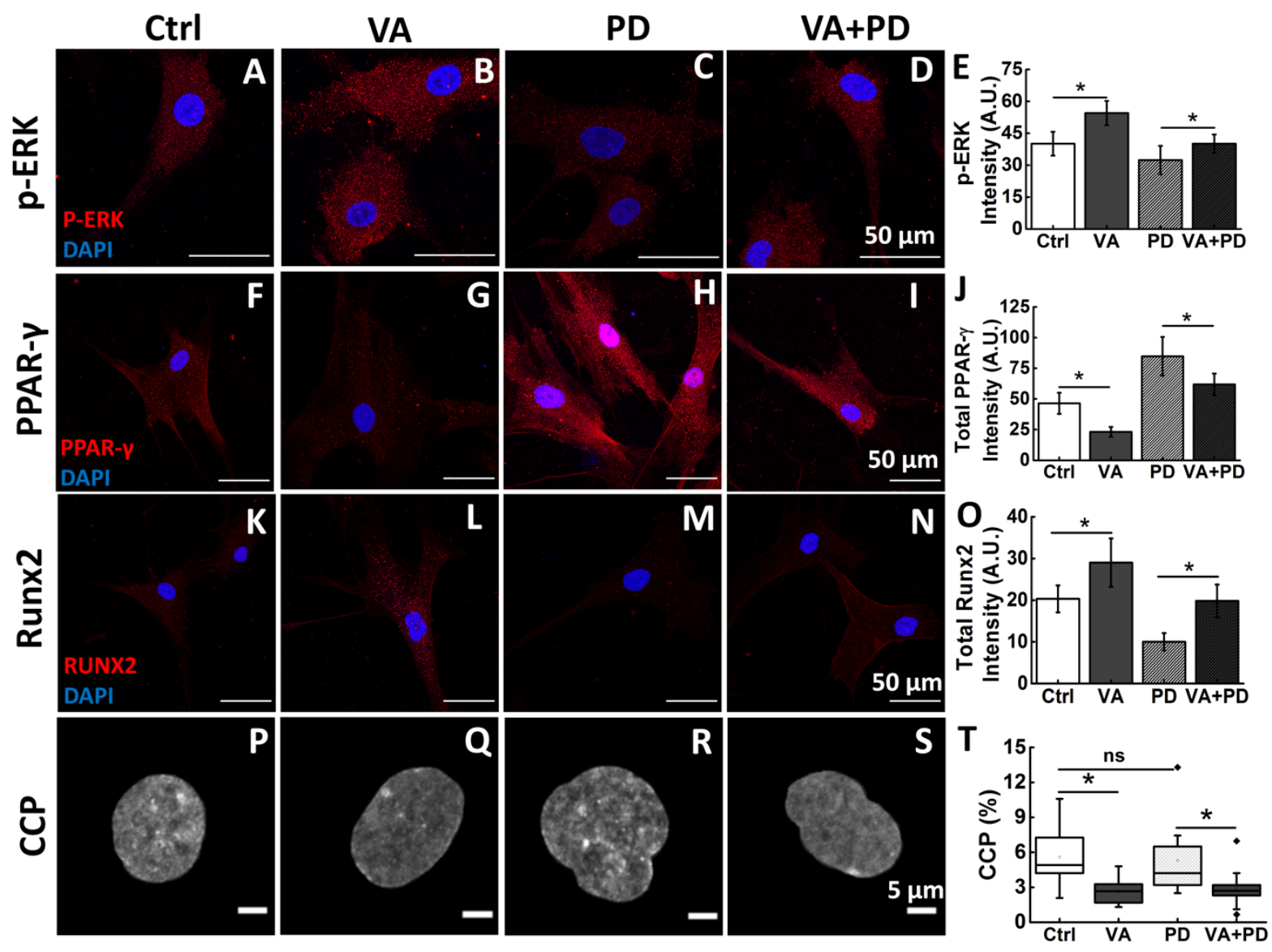

Fig. 6 HDACi increases the expression p-ERK in hMSCs on soft hydrogel : Representative fluorescence images shows p-ERK (red) and nucleus (blue) expression on 3kPa gel in presence of VA, PD98059 and VA+PD98059 with respect to control. Images (F-J) shows PPAR- $\gamma$ (red) expression in presence of VA, 
PD98059 and VA+PD98059 with respect to control. Images (K-O) shows RUNX2 (red) expression in presence of VA, PD98059 and VA+PD98059 with respect to control. Images (P-T) shows chromatin condensation parameter in presence of VA, PD98059 and VA+PD98059 with respect to control. ${ }^{*} p<0.05, N=3 n=25$ Scale Bar: $50 \mu m$

We also checked the effect of $p$-ERK inhibition on PPAR- $\gamma$ and RUNX2 expression. We found that the loss of adipogenic differentiation on soft substrate by VA (Fig. 6G) is restored when simultaneously also treated with PD (Fig. $6 \mathrm{I}$ and J) which by itself promotes adiopgenesis by blocking p-ERK (Fig 6H and J). As expected, an opposite trend was observed for osteogenic differentiation of hMSCs on soft substrates, as shown by the expression of RUNX2 (Fig. 6 K-O). We also checked the chromatin condensation parameter to understand the condensed state of chromatin. We found that VA (2.6 \pm 1.02) significantly reduces chromatin condensation compared to the control $(5.6 \pm 2.26)$. However, application of PD98059 does not have any effect on CCP in presence or absence of VA, indicating phosphorylation of ERK does not interfere with the change in chromatin condensation by acetylation/deacetylation.

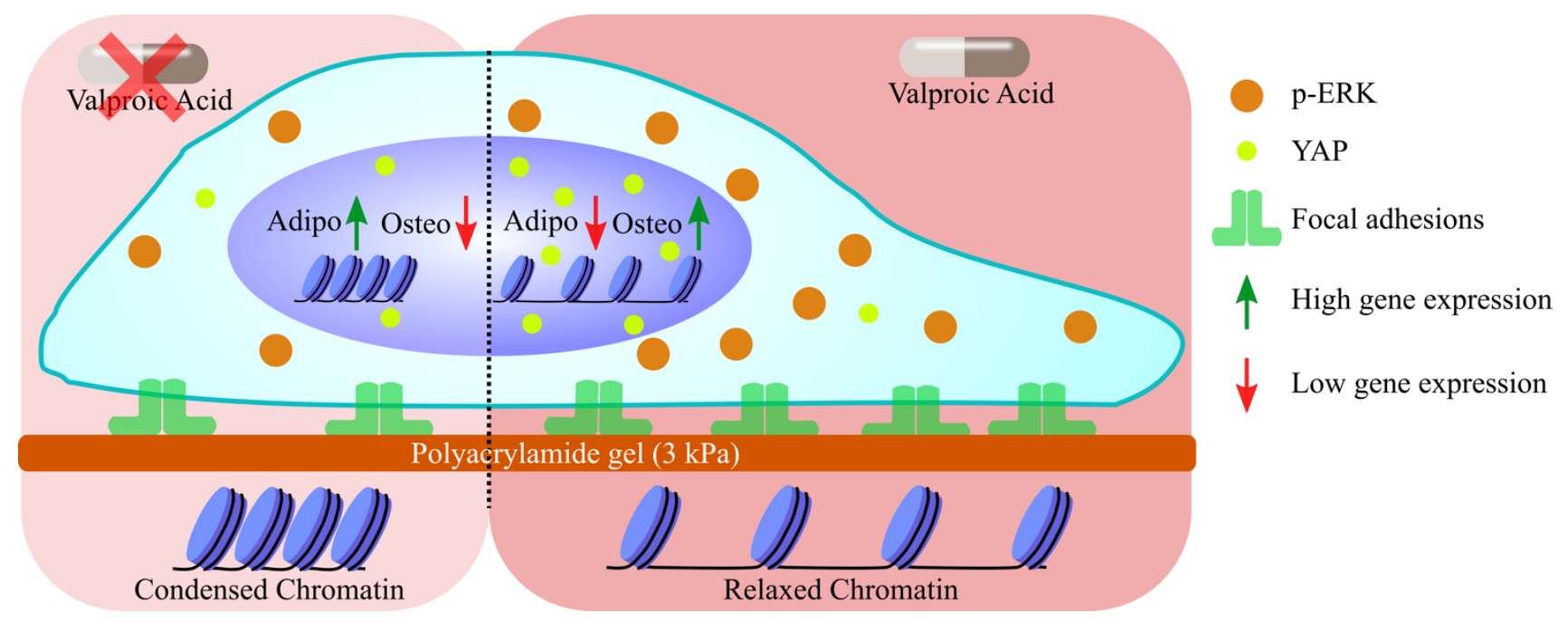

Fig. 7 Schematic showing effect of valproic acid (HDACi) on hMSCs behaviour on soft hydrogel.

\section{Discussion}

In this work, we have found that inhibition of histone deacetylation overrides the effect of substrate stiffness on the behaviour of hMSCs when they are cultured on soft hydrogels (Fig 7). In the presence of VA, a well-established HDACi, hMSCs on soft gels spread more (Fig. 1), generate higher traction (Fig. 
3), and express higher maturation of focal adhesion and formation of stress fibres (Fig. 2). These are some of the critical hallmarks that the cells exhibit when cultured on the rigid substrates but not on the soft substrates. These observations indicate that the effect of substrate rigidity on cellular behaviour can be modulated by modulating chromatin packing. Further, we looked at the mechanosensory protein YAP and found it to localize more in the nucleus as generally seen on stiff substrates (8). Finally, we found that HDACi not only modifies the morphological phenotypes but also control the hMSC differentiation (Fig. 5). While soft substrates promote adipogenic differentiation, in the presence of VA, osteogenic differentiation is preferred. We have further shown that HDACi control differentiation via phosphorylation of Extracellular Regulated Kinase (ERK). If phosphorylation of ERK is inhibited, the chromatin stays in its condensed state but its effect on differentiation disappears and the adipogenic differentiation is restored (Fig. 6).

hMSCs are the popular choice of stem cells in tissue engineering and regenerative medicines due to their tri-lineage differentiation potential $(51,52)$. However, to achieve a desired differentiation outcome, it is important to understand the role of various cues in governing the lineage specification. Matrix stiffness is one of such crucial cue that has been shown to control the differentiation of hMSCs (14). When cultured on soft gel that mimic stiffness of fat tissue, hMSCs commit towards adipogenic lineage. Similarly, when cultured on rigid substrates, osteogenic differentiation is preferred $(11,53)$. Hence, while designing tissue scaffolds, such mechanical control on cellular behaviour needs to be taken into consideration. However, for various other practical reasons, the mechanical properties of the scaffold may not match the desired differentiation outcome. For example, in a bone scaffold, compliant materials might be preferred to faithfully match the defect shape. However, a compliant substrate is unsuitable for osteogenic $(38,42)$ Hence, it is needed to know how such mechanical controls of cellular differentiation can be overwritten by comparing the potency of conflicting cues and understanding the sequence of molecular players.

In this context, we have investigated the effect of histone deacetylase inhibitor HDACi on hMSCS cultured on soft polyacrylamide (PA) hydrogel substrates. HDACi are the group of molecules which are used in chemotherapy. In this work, we have used Valproic Acid which is an FDA approved anti-cancer agent. It has been used in breast cancer, head and neck cancer, cervical cancer etc (54-57). VA has also been used in various neurological disorders such as delirium, agitation, epilepsy (58). Due to its known inhibitory effect in higher concentration ( $\sim \mathrm{mM})(59)$, in this work concentration of VA was kept at its minimal level $(0.5 \mathrm{mM})$.

As already mentioned, soft substrates promote adipogenic differentiation of hMSCs which is associated with epigenetic modification of the chromatin. Recently, Anseth's group have shown that 
substrate stiffness influences chromatin remodelling and epigenetic modification $(28,34)$. hMSCs cultured on stiff substrate have increased histone acetylation due to decreased expression of HDACs and increase expression of HATs leading to more decondensed chromatin state as compared to the cells on soft substrates (34). So, we asked what the cell fate will be if two contradictory signals are provided to the cells, namely mechano-signals from the soft gel which condense the chromatin and promotes adipogenic differentiation and chemical signals from HDACi which decondense opens-the chromatin and promotes osteogenic differentiation. We have found that all the known downstream effect of the soft substrates on hMSCs can be changed by changing acetylation status of the chromatin.

In summary, our results suggest that if histone deacetylation is inhibited then hMSCs on soft gel behave as if they are on a stiff substrate. In other words, effect of mechanosignals can be completely masked by using a chromatin modifier. However, in future it is to be checked if same conclusion can be drawn by activating/inhibiting HATs on soft/stiff substrates. It is also to be seen if other known lineage specifications of hMSCs that is chondrogenic, myogenic, and neurogenic differentiation can be achieved on substrates of different rigidities by simply changing the overall chromatin condensation state by using a cocktail of chromatin modifiers. Overall, the results presented in this paper advance our general understanding of the working of mechanosignals in the context of stem cell differentiation and regenerative medicines.

\section{Author Contribution:}

A.M. and R.J. designed conceptualized and designed the research; A.M. supervised the project. R.J., P.M., P.Y. and V.D. performed research. R.J., P.M., P.Y. and V.D. analyzed the data. R.J. prepared the figures. R.J. and A.M. interpreted the results. R.J., A.M. and P.M wrote the paper. P.M., P.Y., V.D. provided the critical inputs on the manuscript.

\section{Acknowledgement}

This research was funded by Wellcome trust-DBT India Alliance (Project \#IA/E/11/1/500419) and WRCB. RJ thanks DBT for the fellowship (DBT/2016/IIT-B/560). We thank the Bio-AFM and confocal microscopy facility of IIT Bombay. We also thank Dr. Subhajit Sen for discussion and providing sodium butyrate. We thank Dr James P Butler (Harvard Medical School, Department of Medicine, Boston) for his TFM code used for the analysis. 


\section{References}

1. R. G. Wells, The role of matrix stiffness in regulating cell behavior. Hepatology (2008) https:/doi.org/10.1002/hep.22193.

2. T. Yeung, et al., Effects of Substrate Stiffness on Cell Morphology, Cytoskeletal Structure, and Adhesion. 34, 24-34 (2005).

3. F. Martino, A. R. Perestrelo, V. Vinarský, S. Pagliari, G. Forte, Cellular Mechanotransduction: From Tension to Function. Front. Physiol. 9, 824 (2018).

4. F. H. Silver, L. M. Siperko, Mechanosensing and mechanochemical transduction: how is mechanical energy sensed and converted into chemical energy in an extracellular matrix? Crit. Rev. Biomed. Eng. 31, 255-331 (2003).

5. C. C. DuFort, M. J. Paszek, V. M. Weaver, Balancing forces: architectural control of mechanotransduction. Nat. Rev. Mol. Cell Biol. 12, 308-319 (2011).

6. P. Taylor, C. Guilluy, K. Burridge, Nuclear mechanotransduction: Forcing the nucleus to respond. Nucleus, 37-41 (2015).

7. Z. Mohri, A. Del Rio Hernandez, R. Krams, The emerging role of YAP/TAZ in mechanotransduction. J. Thorac. Dis. 9, E507 (2017).

8. S. Dupont, et al., Role of YAP/TAZ in mechanotransduction. Nature 474, 179-183 (2011).

9. S. Dupont, Role of YAP/TAZ in cell-matrix adhesion-mediated signalling and mechanotransduction. Exp. Cell Res. 343, 42-53 (2016).

10. A. Elosegui-Artola, I. Andreu, A. E. M. Beedle, D. Navajas, S. Garcia-Manyes, Force Triggers YAP Nuclear Entry by Regulating Transport across Nuclear Pores (2017) https:/doi.org/10.1016/j.cell.2017.10.008 (December 25, 2021).

11. R. Olivares-Navarrete, et al., Substrate Stiffness Controls Osteoblastic and Chondrocytic Differentiation of Mesenchymal Stem Cells without Exogenous Stimuli. PLoS One 12, e0170312 (2017).

12. L. R. Smith, S. Cho, D. E. Discher, Stem cell differentiation is regulated by extracellular matrix mechanics. Physiology 33, 16-25 (2018).

13. C. Yang, et al., Spatially patterned matrix elasticity directs stem cell fate. Proc. Natl. Acad. Sci. U. S. A. 113, E4439-E4445 (2016).

14. A. J. Engler, S. Sen, H. L. Sweeney, D. E. Discher, Matrix Elasticity Directs Stem Cell Lineage Specification. Cell 126, 677-689 (2006).

15. J. Oliver-De La Cruz, et al., Substrate mechanics controls adipogenesis through YAP phosphorylation by dictating cell spreading. Biomaterials 205, 64-80 (2019).

16. S. K. Kureel, et al., Soft substrate maintains proliferative and adipogenic differentiation potential of human mesenchymal stem cells on long-term expansion by delaying senescence. Biol. Open 8 (2019).

17. M. B. Meyer, N. A. Benkusky, B. Sen, J. Rubin, J. W. Pike, Epigenetic Plasticity Drives Adipogenic and Osteogenic Differentiation of Marrow-derived Mesenchymal Stem Cells. J. 
Biol. Chem. 291, 17829-17847 (2016).

18. M. J. Boland, K. L. Nazor, J. F. Loring, Epigenetic regulation of pluripotency and differentiation. Circ. Res. 115, 311 (2014).

19. J. Kazakevych, S. Sayols, B. Messner, C. Krienke, N. Soshnikova, Dynamic changes in chromatin states during specification and differentiation of adult intestinal stem cells. Nucleic Acids Res. 45, 5770-5784 (2017).

20. L. Verdone, M. Caserta, E. Di Mauro, Role of histone acetylation in the control of gene expression. https://doi.org/10.1139/o05-041 83, 344-353 (2011).

21. A. J. Bannister, T. Kouzarides, Regulation of chromatin by histone modifications. Cell Res. 21, 381-395 (2011).

22. D. Y. Lee, J. J. Hayes, D. Pruss, A. P. Wolffe, A positive role for histone acetylation in transcription factor access to nucleosomal DNA. Cell 72, 73-84 (1993).

23. A. J. Bannister, T. Kouzarides, Regulation of chromatin by histone modifications. Cell Res. 2011213 21, 381-395 (2011).

24. A. Dudakovic, et al., Histone Deacetylase Inhibition Promotes Osteoblast Maturation by Altering the Histone H4 Epigenome and Reduces Akt Phosphorylation *. J. Biol. Chem. 288, 28783-28791 (2013).

25. Q. Tian, S. Gao, X. Zhou, L. Zheng, Y. Zhou, Histone Acetylation in the Epigenetic Regulation of Bone Metabolism and Related Diseases. Stem Cells Int. 2021 (2021).

26. J. Shen, et al., Transcriptional Induction of the Osteocalcin Gene During Osteoblast Differentiation Involves Acetylation of Histones H3 and H4. Mol. Endocrinol. 17, 743-756 (2003).

27. F. M. Pérez-Campo, J. A. Riancho, Epigenetic Mechanisms Regulating Mesenchymal Stem Cell Differentiation. Curr. Genomics 16, 368 (2015).

28. A. R. Killaars, C. J. Walker, K. S. Anseth, Nuclear mechanosensing controls MSC osteogenic potential through HDAC epigenetic remodeling. Proc. Natl. Acad. Sci. 117, 21258-21266 (2020).

29. J. Y. Eung, J. J. Chung, S. C. Sung, H. K. Kang, B. K. Jae, Down-regulation of histone deacetylases stimulates adipocyte differentiation. J. Biol. Chem. 281, 6608-6615 (2006).

30. T. K. Chatterjee, et al., Histone deacetylase 9 is a negative regulator of adipogenic differentiation. J. Biol. Chem. 286, 27836-27847 (2011).

31. A. Satoh, et al., Valproic acid promotes differentiation of adipose tissue-derived stem cells to neuronal cells selectively expressing functional $\mathrm{N}$-type voltage-gated $\mathrm{Ca} 2+$ channels. Biochem. Biophys. Res. Commun. 589, 55-62 (2022).

32. P. Choudhary, A. Gupta, S. Singh, Therapeutic Advancement in Neuronal Transdifferentiation of Mesenchymal Stromal Cells for Neurological Disorders. J. Mol. Neurosci. 2020715 71, 889901 (2020).

33. S. Rashid, et al., Effect of valproic acid on the hepatic differentiation of mesenchymal stem cells in 2D and 3D microenvironments. Mol. Cell. Biochem. 476, 909-919 (2021).

34. A. R. Killaars, et al., Extended exposure to stiff microenvironments leads to persistent chromatin remodeling in human mesenchymal stem cells. Trans. Annu. Meet. Soc. Biomater. 
Annu. Int. Biomater. Symp. 40, 670 (2019).

35. M. Göttlicher, Valproic acid: an old drug newly discovered as inhibitor of histone deacetylases. Ann. Hematol. 83 Suppl 1 (2004).

36. C. J. Phiel, et al., Histone Deacetylase Is a Direct Target of Valproic Acid, a Potent Anticonvulsant, Mood Stabilizer, and Teratogen *. J. Biol. Chem. 276, 36734-36741 (2001).

37. M. L. S. Mello, Sodium Valproate-Induced Chromatin Remodeling. Front. Cell Dev. Biol. 9 (2021).

38. T. Zhang, et al., Regulating osteogenesis and adipogenesis in adipose-derived stem cells by controlling underlying substrate stiffness. J. Cell. Physiol. 233, 3418-3428 (2018).

39. J. H. Hwang, et al., Extracellular matrix stiffness regulates osteogenic differentiation through MAPK activation. PLoS One 10, 1-16 (2015).

40. J. R. Tse, A. J. Engler, Preparation of Hydrogel Substrates with Tunable Mechanical Properties https:/doi.org/10.1002/0471143030.cb1016s47 (December 29, 2021).

41. R. Vishavkarma, et al., Role of actin filaments in correlating nuclear shape and cell spreading. PLoS One 9 (2014).

42. S. K. Kureel, et al., Soft substrate maintains proliferative and adipogenic differentiation potential of human mesenchymal stem cells on long-term expansion by delaying senescence. Biol. Open 8 (2019).

43. P. S. Mathieu, E. G. Loboa, Cytoskeletal and Focal Adhesion Influences on Mesenchymal Stem Cell Shape, Mechanical Properties, and Differentiation Down Osteogenic, Adipogenic, and Chondrogenic Pathways. Tissue Eng. Part B. Rev. 18, 436 (2012).

44. C. M. Kraning-Rush, S. P. Carey, J. P. Califano, B. N. Smith, C. A. Reinhart-King, The role of the cytoskeleton in cellular force generation in 2D and 3D environments. Phys. Biol. 8, 015009 (2011).

45. B. R. Sarangi, et al., Coordination between Intra- and Extracellular Forces Regulates Focal Adhesion Dynamics. Nano Lett. 17, 399-406 (2017).

46. J. P. Califano, C. A. Reinhart-King, Substrate Stiffness and Cell Area Predict Cellular Traction Stresses in Single Cells and Cells in Contact. Cell. Mol. Bioeng. 3, 68 (2010).

47. S. J. Han, K. S. Bielawski, L. H. Ting, M. L. Rodriguez, N. J. Sniadecki, Decoupling Substrate Stiffness, Spread Area, and Micropost Density: A Close Spatial Relationship between Traction Forces and Focal Adhesions. Biophys. J. 103, 640 (2012).

48. B. L. Doss, et al., Cell response to substrate rigidity is regulated by active and passive cytoskeletal stress https:/doi.org/10.1073/pnas.1917555117/-/DCSupplemental (December 25, 2021).

49. J. H. Hwang, et al., Extracellular Matrix Stiffness Regulates Osteogenic Differentiation through MAPK Activation. PLoS One 10, e0135519 (2015).

50. P. E. Farahani, et al., Substratum stiffness regulates Erk signaling dynamics through receptorlevel control. Cell Rep. 37, 110181 (2021).

51. Y. Han, et al., Mesenchymal Stem Cells for Regenerative Medicine. Cells 8, 886 (2019).

52. M. F. Pittenger, et al., Mesenchymal stem cell perspective: cell biology to clinical progress. $n p j$ Regen. Med. 201941 4, 1-15 (2019). 
53. C. B. Khatiwala, P. D. Kim, S. R. Peyton, A. J. Putnam, ECM compliance regulates osteogenesis by influencing MAPK signaling downstream of RhoA and ROCK. J. Bone Miner. Res. 24, 886898 (2009).

54. H. Heers, J. Stanislaw, J. Harrelson, M. W. Lee, Valproic acid as an adjunctive therapeutic agent for the treatment of breast cancer. Eur. J. Pharmacol. 835, 61-74 (2018).

55. C. P. Gan, et al., Valproic acid: growth inhibition of head and neck cancer by induction of terminal differentiation and senescence. Head Neck 34, 344-353 (2012).

56. C. Tsai, et al., Valproic acid suppresses cervical cancer tumor progression possibly via activating Notch1 signaling and enhances receptor-targeted cancer chemotherapeutic via activating somatostatin receptor type II. Arch. Gynecol. Obstet. 288, 393-400 (2013).

57. F. Caponigro, et al., Phase II clinical study of valproic acid plus cisplatin and cetuximab in recurrent and/or metastatic squamous cell carcinoma of Head and Neck-V-CHANCE trial. BMC Cancer 16, 1-10 (2016).

58. M. Romoli, et al., Valproic Acid and Epilepsy: From Molecular Mechanisms to Clinical Evidences. Curr. Neuropharmacol. 17, 926 (2019).

59. H. Cha, J. Lee, H. H. Park, J. H. Park, Direct Conversion of Human Fibroblasts into Osteoblasts Triggered by Histone Deacetylase Inhibitor Valproic Acid. Appl. Sci. 2020, Vol. 10, Page 7372 10, 7372 (2020).

\section{SUPPLEMENTARY INFORMATION}

Table S1

$\begin{array}{llcc}\text { Acrylamide from } & \text { Bis-acrylamide from } & \text { Water } & E \pm \text { St. Dev. } \\ 40 \% \text { stock solution }(\mathrm{ml}) & 2 \% \text { Stock solution }(\mathrm{ml}) & (\mathrm{ml}) & (\mathrm{kPa})\end{array}$
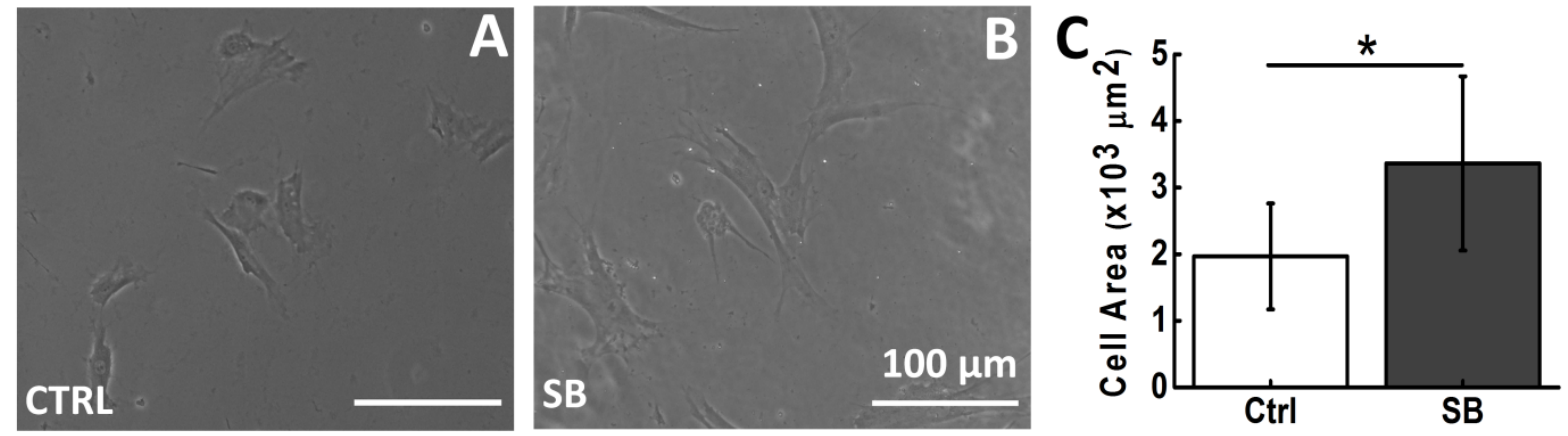
Fig. S1. HDACi increases cell spread area on soft hydrogel. Representative images of hMSCS cultured on $3 \mathrm{kPa}$ gel (A) without SB and (B) with SB. Graph (C) show quantitative analysis of change in projected cell area on $3 \mathrm{kPa}$ gels with and without $\mathrm{SB} .{ }^{*} p<0.001 \mathrm{n}>50$ cells, scale bar: $100 \mu \mathrm{m}$.
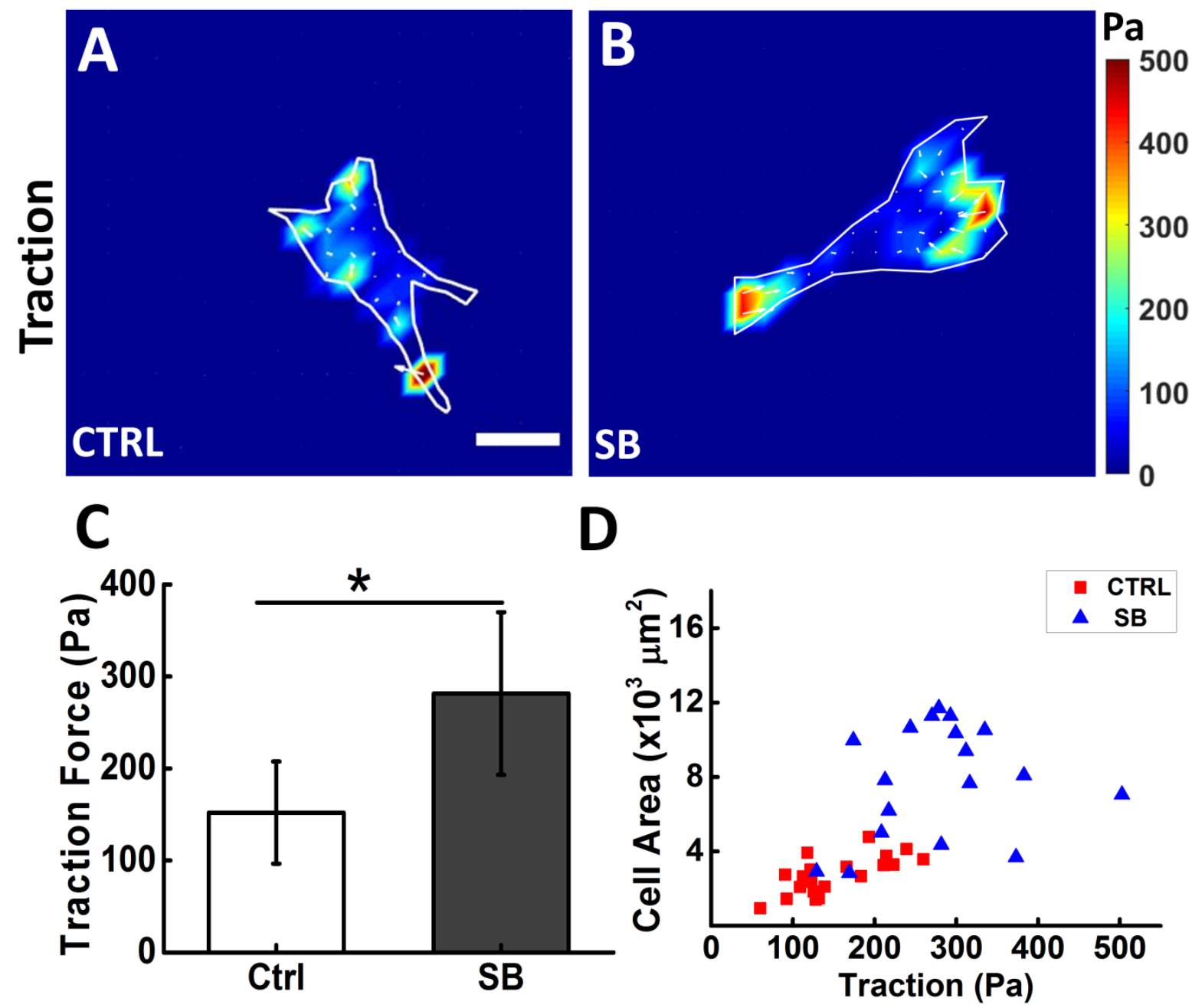

Fig. S2. HDACi increases hMSCs traction on soft hydrogel. Heat map of hMSCs traction stress (A) without SB, (B) with SB. Colours corresponds to magnitude of stresses as indicated in the colour bar. Graph (C) shows quantitative analysis of traction force on $3 \mathrm{kPa}$ gel with and without SB (Ctrl). Scatter plot (D) shows positive correlation between cell spread area and cellular traction. ${ }^{*} p<0.001 \mathrm{n}=18$ cells scale bar: $100 \mu \mathrm{m}$. 

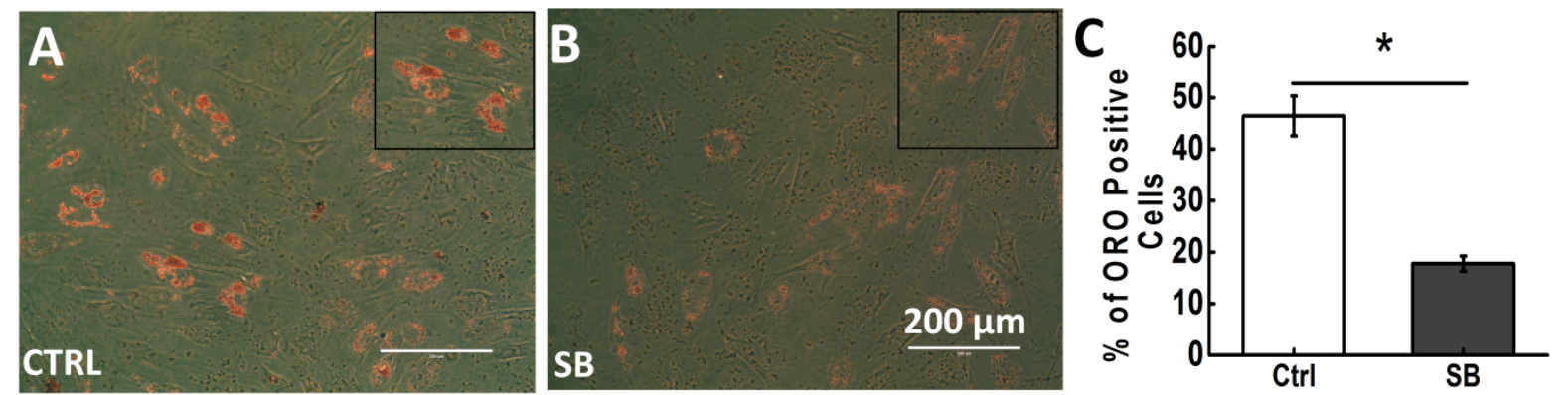

Fig. S3. HDACi inhibit adipogenesis of hMSCs cultured on soft hydrogel. Representative images of Oil Red O staining on $3 \mathrm{kPa}$ gel without SB (A) and with SB (B). The insets are the magnified local image to show Oil Red $O$ formation. Graph (C) shows quantitative analysis of change in percentage of ORO positive cells with and without SB. ${ }^{*} p<0.001$ scale bar: $100 \mu \mathrm{m}$. 
bioRxiv preprint doi: https://doi.org/10.1101/2022.01.04.474891; this version posted January 4, 2022. The copyright holder for this preprint (which was not certified by peer review) is the author/funder, who has granted bioRxiv a license to display the preprint in perpetuity. It is made available under aCC-BY-ND 4.0 International license. 